\title{
Characterization of microRNA in bovine in vitro culture media associated with embryo quality and development
}

\author{
Jenna Kropp and Hasan Khatib ${ }^{1}$ \\ Department of Animal Sciences, University of Wisconsin, Madison 53706
}

\begin{abstract}
Dairy cattle fertility has declined over time due to factors including reduced fertilization and early embryonic loss. To counter fertility problems and better study preimplantation embryonic development, in vitro production systems have been developed. These systems largely assess embryos based on their morphology, which is not a strong indicator of developmental potential. Currently, no biomarkers can be used to noninvasively survey an embryo's potential in terms of its development and ability to establish a pregnancy. Thus, the objective of this study was to characterize and identify microRNA (miRNA) in culture media of embryos of differing developmental competence for future development as noninvasive biomarkers of embryo quality. The MiRNA sequencing of media conditioned by blastocyst and degenerate (those that failed to develop from the morula to blastocyst stage) embryos, revealed 11 differentially expressed miRNA; all were higher in concentration in degenerate conditioned media. Differential expression of mature microRNA (miR)-24, miR-191, and miR-148a was further validated using quantitative real-time PCR. Functional analysis of miR-24 revealed that addition of a mimic miRNA to culture media of morulae embryos resulted in a $27.3 \%$ decrease in development to the blastocyst stage. Furthermore, expression of miR-24 was 44.29-fold higher in blastocysts cultured with a miR-24 mimic compared with control blastocysts. Interestingly, the expression of $C D K N 1 b$, a target gene of miR-24 was repressed in embryos grown in the presence of the miRNA mimic. Mimic supplementation experiments suggest that miRNA are taken up by the embryo and that extracellular miRNA affect embryonic development. Overall, identification of a rich extracellular milieu in conditioned media sets the framework for future studies to determine the long-term predictive ability of embryo-based miRNA biomarkers on pregnancy outcome.
\end{abstract}

Received February 25, 2015.

Accepted May 13, 2015.

${ }^{1}$ Corresponding author: hkhatib@wisc.edu
Key words: small RNA sequencing, embryonic development, media, microRNA, in vitro fertilization

\section{INTRODUCTION}

Infertility has become a challenge in many mammalian species. In humans, about $15 \%$ of couples fail to conceive within the course of a year of unprotected sex. Within the United States, $10.9 \%$ of women ages 15 to 44 yr have impaired fecundity and about $6 \%$ are infertile (Chandra et al., 2014). A decline in fertility over the last 40 to $50 \mathrm{yr}$ has also been observed in dairy cattle, as producers increased selection intensity for milk production traits (Lucy, 2001). In dairy cattle multiple factors contribute to the decline in fertility, including high early and late embryonic loss (Santos et al., 2004; Diskin and Morris, 2008). Conception rates in the 1950 s were reported to be about $55 \%$ for AI cows at observed estrus, and by 1990 the rate declined to about 35\% (Lucy, 2001; Diskin et al., 2012). Reproductive inefficiency is costly to dairy producers. Thus, it is necessary to better understand mechanisms underlying embryo development and discover molecular markers to identify embryos that will successfully or unsuccessfully establish a pregnancy to improve fertility in both dairy cattle and humans.

Assisted reproductive technologies have become well developed and used to overcome some of the challenges of infertility. In vitro fertilization (IVF) to produce embryos is advantageous because it bypasses challenges associated with fertilization, allows for widespread use of gametes from genetically superior animals, and also establishes a system to better study the window of early embryonic development. However, assessment of embryo quality and potential of in vivo and IVF embryos, in both humans and cattle, is largely based on morphology, which is often not indicative of the embryo's ability to establish a pregnancy (Van Soom et al., 2003). Whereas embryos are often of similar morphology, the underlying genetics, such as the transcriptomes, can be strikingly different. A recent study demonstrated that 793 genes were differentially expressed in embryos of similar morphology but differed by whether they were 
produced in an in vivo or in vitro production system (Driver et al., 2012). Indeed, the pregnancy rate of recipients carrying an in vitro-produced embryo is lower, and there is increased risk for abortion and stillbirth compared with recipient cows carrying an in vivo-produced embryo (Farin et al., 2006). Thus, a need exists for a better noninvasive method for efficient selection of embryos based on their genetic potential and ability to establish a pregnancy.

MicroRNA (miRNA) biomarkers have recently been identified for detection of several pathologies in humans. Indeed, Mitchell et al. (2008) demonstrated that miRNA secreted from prostate cancer cells into the blood could be detected in a patient's serum sample, offering a noninvasive method for diagnosing cancer. The presence of miRNA in the extracellular environment has recently been reported in many bodily fluids, including serum, saliva, urine, and placental fluid (Valadi et al., 2007; Chen et al., 2008; Mitchell et al., 2008; Wang et al., 2010; Turchinovich et al., 2011; Rayner and Hennessy, 2013; Xu et al., 2013a). Studies have demonstrated the extreme stability of miRNA in serum as they withstand freezing, thawing, and $\mathrm{pH}$ changes (Chen et al., 2008; Mitchell et al., 2008). In the extracellular environment, miRNA are predominantly bound to proteins such as argonaute 2 (Ago2) and nucleophosmin 1 (NPM1), which provide stability, though a fraction is also contained within exosomes (Valadi et al., 2007; Wang et al., 2010; Turchinovich et al., 2011). Secretion of miRNA into the extracellular environment is cell-specific, as evident by identification of tumor cellspecific miRNA in serum (Chen et al., 2008; Mitchell et al., 2008), which allows for development of biomarkers for a wide range of cell types and tissues.

Mature miRNA are short, noncoding RNA (18-22 nucleotides in length) that play a role in nearly every cellular process including cell proliferation, differentiation, metabolism, apoptosis, and cell signaling (Filipowicz et al., 2008; Wang et al., 2013). In the cytoplasm of cells, these small RNA molecules are assembled into RNAinduced silencing complexes where the guide strand of the miRNA binds to the $3^{\prime}$ UTR region of its target mRNA to either degrade or block translation of the mRNA, thereby blocking protein synthesis (Filipowicz et al., 2008). Imperfect binding to a miRNA's target mRNA allows for one miRNA to bind to a vast array of mRNA targets, increasing the regulatory potential of a single miRNA (Ambros, 2004; Farh et al., 2005; Lim et al., 2005). The MiRNA expression is precisely regulated in a developmental stage and tissue-specific manner in all cell types, including embryos (Bartel, 2004; Farh et al., 2005; Lim et al., 2005). Therefore, miRNA expression in the embryo is of great importance, and dysregu- lation of key miRNA could consequently alter the genes regulating embryonic development.

Identification of differentially expressed miRNA in the media between embryos of varying competence could be used as prognostic molecular markers. In a proof-of-concept study by Kropp et al. (2014), miRNA were detected in human and bovine IVF culture media. Similarly, Rosenbluth et al. (2014) also detected miRNA in human IVF culture media and differential miRNA expression was correlated with pregnancy outcome and chromosomally abnormal embryos. These studies are limited in the number of miRNA detected, as they studied a few candidate miRNA (Kropp et al., 2014) or used a heterologous array for detection of miRNA (Rosenbluth et al., 2014). Further investigation utilizing miRNA-sequencing (miRNA-seq) technology would allow for more thorough characterization of both known and novel miRNA within IVF culture media.

The miRNA expression within embryos differs between embryos of varying developmental competence, and thus the extracellular spectra of miRNA are likely to reflect these differences as well. We hypothesized that, through miRNA-seq, differentially expressed miRNA would be identified in the extracellular IVF culture media conditioned by embryos of extreme differing developmental potential and that those miRNA may play a role in embryonic development. To test our hypothesis, the extracellular milieu of conditioned media was characterized by miRNA-seq to determine whether miRNA were differentially expressed. Then functional analysis by supplementation of a synthetic miRNA (a mimic miRNA similar in structure to endogenous miRNA) was added to the medium of morula stage embryos to assess the effect of extracellular miRNA on embryo development.

\section{MATERIALS AND METHODS}

\section{In Vitro Production of Embryos}

As previously described in the study of Kropp et al. (2014), 3 replicates of embryos and culture media were procured utilizing an IVF system. Here, in brief, ovaries were obtained from a local slaughterhouse and follicles of 2 to $10 \mathrm{~mm}$ were aspirated. Oocytes were washed twice in Vigro TL-Hepes (Bioniche, Pullman, WA) buffer supplemented with $3 \%$ BSA, sodium pyruvate, and gentamicin. Washed cumulus-oocyte complexes were transferred in groups of 10 into a $50-\mu \mathrm{L}$ drop of M-199 medium supplemented with sodium pyruvate, gonadotropins (FSH and LH), estradiol, gentamicin, and 10\% fetal bovine serum. Cumulus oocyte complexes were 
matured for $24 \mathrm{~h}$, at which point they were washed in supplemented Vigro TL-Hepes (Bioniche) and transferred in cohorts of 10 into a $44-\mu \mathrm{L}$ drop of IVF-TL medium (Millipore, Phillipsburg, NJ) supplemented with FA-free BSA, sodium pyruvate, and gentamicin. Sperm were prepared using the swim-up protocol as described by Parrish et al. (1995), where the final concentration was adjusted to 1 million sperm $/ \mathrm{mL}$. Additionally, 2 $\mu \mathrm{L}$ of each heparin (where the final concentration was $2.0,5.0$, or $10.0 \mu \mathrm{g} / \mathrm{mL}$, respectively, depending on the characterized response for each bull to capacitate) and a PHE cocktail (40\% saline, 25\% $1 \mathrm{mM}$ penicillin, $25 \% 2 \mathrm{~m} M$ hypotaurine, and $10 \% 250 \mu M$ epinephrine) were added to each fertilization drop. Oocytes were coincubated with sperm for $24 \mathrm{~h}$; the time of fertilization began at day zero.

Following $24 \mathrm{~h}$ of coincubation of sperm and oocytes, presumptive zygotes were stripped of their cumulus cells and washed in supplemented Vigro TL-Hepes (Bioniche). The presumptive zygotes were then placed in cohorts of 25 per $50-\mu \mathrm{L}$ drops of synthetic oviductal fluid (SOF) media (Millipore) supplemented with FAfree BSA, sodium pyruvate, AA, and gentamicin to be cultured until d 5 . On d 5 , embryos were morphologically assessed and those that showed characteristics of a compacted morula were transferred to a fresh 50$\mu \mathrm{L}$ drop of SOF media lacking BSA supplementation, as serum albumins have been shown to be carriers of miRNA, and cultured individually.

On d 8 of development, embryos were again morphologically assessed for characteristics of either a blastocyst or a late degenerate. Mid- and expanded blastocysts of stages 6 and 7 and of quality grades ranging from 1 to 3, according to the IETS standards described by Bo and Mapletoft (2013),were collected and pooled together. Previously our laboratory revealed that the correlation of gene expression between late degenerate or growth-retarded embryos and blastocyst embryos is high $(\mathrm{r}=0.986)$, yet the degenerates fail to undergo differentiation and development blastocoel cavity (Huang et al., 2010). The degenerate embryos allow for study of genes potentially underlying the development to the blastocyst stage and embryo quality. Degenerate embryos were collected here and pooled together. Both the media (conditioned by a single embryo) and the embryos were collected in parallel and pooled into respectively either the blastocyst or degenerate sample for a given IVF experiment. Control SOF media drops (lacking BSA supplementation) that were cultured within the same petri dish plate as drops containing an embryo were also collected. One pool was generated for each type of embryo in 3 IVF replicates for miRNA-seq utilizing a separate sire for each pool (see
Supplementary Table S1 for pool design; http://dx.doi. org/10.3168/jds.2015-9510).

\section{MiRNA Extraction from Culture Media}

Extraction of miRNA from culture media samples was carried out using a cell-free specific kit, the miRNeasy Serum/Plasma kit (Qiagen, Germantown, MD). The input volume for extraction was $200 \mu \mathrm{L}$ for each pooled sample. To meet miRNA-seq input requirements, multiple extractions of each sample were carried out where the eluted miRNA samples from each extraction were pooled together. The RNA was quantified using NanoDrop ND-1000 spectrophotometer (NanoDrop Technologies, Wilmington, DE). Following 3 extractions utilizing a total of $600 \mu \mathrm{L}$ per pooled media sample, total RNA isolated for the media pools ranged from 780 to $1,490 \mathrm{ng}$ for blastocyst media, 730 to $1,090 \mathrm{ng}$ for degenerate media, and 610 to $950 \mathrm{ng}$ for control SOF media pools. Samples were sent for library preparation and sequencing by LC Sciences (Houston, TX).

\section{Small RNA Library Construction and Deep Sequencing}

Small RNA library preparation was carried out by LC Sciences using the Illumina TruSeq Small-RNA Preparation kit (Illumina, San Diego, CA) following the kit's guidelines using equivalent amounts of RNA per sample. The cDNA libraries generated were quality checked via an Agilent Bioanalyzer (Agilent, Santa Clara, CA) and then clustered on Illumina's Cluster Station followed by sequencing on an Illumina GAIIx instrument.

\section{Small RNA-Sequencing Data Analysis}

Sequencing data were analyzed using the LC Sciences proprietary pipeline. The raw sequenced reads were extracted and filtered to remove impure sequences, which included removal of sequences that did not contain a $3^{\prime}$ adaptor and those that were less than 15 bases after the $3^{\prime}$ adaptor cut. Raw reads which were between 15 and 32 bases in length and of the same sequence were clustered into families deemed unique sequences.

Unique sequences were mapped to pre-miRNA (denoted as mir) and mature miRNA (denoted as miR) sequences listed in miRbase (http://www.mirbase.org/) of bovine or comparatively to other selected mammalian species. The mapping strategy is depicted in Supplementary Figure S1 (http://dx.doi.org/10.3168/ jds.2015-9510). In brief, reads were mapped to miRbase and also to the bovine genome (bovine reference 
assembly UMD 3.1) using the alignment software Bowtie (Johns Hopkins University; http://bowtie-bio. sourceforge.net/index.shtml) allowing for 1 mismatch. A proprietary pipeline script (ACGT101-miR, LC Sciences) was used to analyze the sequencing data. Mappable sequences were divided into 2 groups those that were mapped to selected mir and mapped to the bovine genome (group 1) or those that were not mapped to the bovine genome (groups 2 and 3). Group 1 was split into 3 subgroups. Group 1a was those that were mapped to selected miR in miRbase, were mapped to the bovine genome, and are known miR of the bovine species. Group $1 \mathrm{~b}$ sequences mapped to selected miR in miRbase, were mapped to the bovine genome, and are known miR in other selected mammalian species. Group 1c includes sequences that mapped not only to the location of known miR, but also to other loci within the bovine genome. Group $2 \mathrm{miR}$ include both group $2 \mathrm{a}$ and $2 \mathrm{~b}$ sequences that mapped to miR of selected species, but did not map to the bovine genome. Group $2 \mathrm{a}$ included those that the extended sequences at that position could potentially form hairpins, whereas in group $2 \mathrm{~b}$ the extended sequences at the mapped position could not form hairpins. Group $3 \mathrm{a}$ miR were those unique sequences that mapped to miR of selected species, but the miR did not map to the bovine genome. Criteria were similar for group $3 \mathrm{~b}$ in that miR did not map to the bovine genome; however, though the miR mapped to mir in miRbase, they did not map to the selected mammalian species chosen for analysis. Those that were unmapped to selected miR in miRbase were further divided into 2 subgroups, group 4a and group $4 \mathrm{~b}$, where both groups did not map to selected miR of bovine but did map to the genome. Group 4a sequences were in a location where the extended sequences were likely to form a hairpin, whereas group $4 \mathrm{~b}$ extended sequences were not likely to form hairpins. The program UNAFold (The RNA Institute, University at Albany, New York) was used to calculate the secondary structure such as the likelihood to form hairpins from the precursor sequence.

\section{Sample Comparison and Statistical Analysis of Sequencing Data}

From each sample, the total number of reads was tracked and normalized by dividing the number of reads by a library size parameter of the corresponding sample. The library size parameter is a median value of the ratio between the counts of a specific sample and a pseudo-reference sample. A count number in the pseudo-reference sample is the count geometric mean across all samples:

$$
S_{j}=\operatorname{median}_{j}\left(\frac{c_{i j}}{\left(\prod_{k=1}^{m} c_{i k}\right)^{1 / m}}\right),
$$

where $S_{j}$ is the library size parameter; $c_{i j}$ is the count number of sequence $i$ sample $j ; m$ is the total number of samples involved; and $k$ is a sample index used for the geometric mean of counts across all samples. A paired $t$-test was carried out across the data from samples of the 3 replicates of sequenced media where significance was set at $P<0.05$.

\section{Real-Time Quantitative PCR for miRNA Gene Expression Validation}

Of the significant miRNA found to be differentially expressed, 5 were chosen to be validated technically by real-time quantitative (qRT)-PCR and biologically in 3 additional IVF replicates. Extracted miRNA for each sample was reverse transcribed into cDNA using a Miscript II RT kit (Qiagen). The HiSpec buffer (Qiagen) was used per kit recommendations for subsequent quantification of mature miRNA. For quantification of gene expression, the reactions were carried out using the miScript SYBR Green Kit (Qiagen). For each miRNA, a specific primer for its mature sequence was designed and obtained from Qiagen (Supplementary Table S2; http://dx.doi.org/10.3168/jds.2015-9510). The qRT-PCR reactions were carried out in a Bio-Rad iCycler real-time PCR machine (Bio-Rad, Hercules, $\mathrm{CA}$ ) with the following cycling conditions: $95^{\circ} \mathrm{C}$ for 15 min followed by 40 cycles of $94^{\circ} \mathrm{C}$ for $15 \mathrm{~s}, 55^{\circ} \mathrm{C}$ for 30 $\mathrm{s}$, and $70^{\circ} \mathrm{C}$ for $30 \mathrm{~s}$. The threshold for detection was set as a cycle threshold $(\mathrm{Ct})<34$. Data were then analyzed using a paired $t$-test on the $\Delta \mathrm{Ct}$ values for each miRNA and graphically represented as the mean fold change in gene expression \pm standard error using the $2^{-\Delta \Delta \mathrm{Ct}}$ method by Livak and Schmittgen (2001).

\section{MiRNA Mimic Supplementation to Embryos}

The IVF embryos were procured as previously mentioned, however, on d 5 , a cohort of 5 to 15 morulae were transferred to a fresh drop of SOF media lacking BSA supplementation. A miRNA mimic of miR-24 was obtained from Qiagen. The mimic is a synthetically produced double-stranded RNA fragment of similar sequence and nature as endogenous mature miRNA. The mimic was reconstituted in water and was added to the medium at a final concentration of $1 \mu M$. The concentration was adjusted based on the manufacturer's 
recommendations for cell culture experiments. Additionally, a cohort of 5 to 15 morulae were transferred to a drop where nuclease-free water (vehicle of mimic) was added at the same volume as the mimic and cultured as control embryos. On d 8, embryos were morphologically assessed and blastocyst and degenerate embryos in each experimental group (mimic or control) were counted. Embryo development was calculated as the percentage of morulae that reached the blastocyst stage. Development was observed in 4 IVF replicates, and blastocyst and degenerate embryos were collected for each control and mimic experiment group from 3 of these replicates for expression analysis.

\section{Gene Expression Analysis of miR-24 and $m R N A$ Targets in Embryos}

To assess miR-24 expression in mimic supplemented and control embryos, an Ambion RNAqueous-Micro Total RNA Isolation kit (Life Technologies, Austin, Texas) was used to isolate total RNA from embryos following the kit recommendation to add 1.25 volumes of ethanol to capture small RNA. Then total RNA was reverse transcribed using the Miscript II RT kit (Qiagen). The HiFlex buffer (Qiagen) was used for subsequent quantification of both mature miRNA and mRNA. Each cDNA reaction was diluted with water at a ratio of 1:5 and combined with $2 \times$ Quantitect SYBR green (Qiagen), water, and either a universal primer and miR-24 primer assay or the primer for the housekeeping gene, GAPDH. The GAPDH gene was chosen as an internal control following the stability test described by Vandesompele et al. (2002). In our previous studies of embryo samples (Huang et al., 2010; Zhang et al., 2012; Driver et al., 2013), the expression of GAPDH showed the smallest relative stability value $\mathrm{M}$ in comparison to $R P L P 0$ (ribosomal protein, large, $\mathrm{P} 0$ ) and $A C T B$ (actin, $\beta$ ). The $\mathrm{M}$ value is the average of the pair-wise variation when compared with the other control genes across the examined samples (Vandensompele et al., 2002). Primer sequences are listed in Supplementary Table S2 (http://dx.doi.org/10.3168/jds.2015-9510). The qRT-PCR reactions were carried out in a Bio-Rad iCycler with the following conditions: $95^{\circ} \mathrm{C}$ for $15 \mathrm{~min}$, followed by 40 cycles of $94^{\circ} \mathrm{C}$ for $15 \mathrm{~s}, 55^{\circ} \mathrm{C}$ for $30 \mathrm{~s}$, and $70^{\circ} \mathrm{C}$ for $30 \mathrm{~s}$.

Additionally, embryos were tested for expression of a miR-24 target mRNA, cyclin-dependent kinase inhibitor 1b $(C D K N 1 b)$. For gene expression analysis, the extracted RNA from embryos described previously was reverse transcribed using the iScript cDNA synthesis kit (Bio-Rad). Real-time PCR reactions were carried out using the iQSYBR Green Supermix kit (Bio-Rad). Primers for qRT-PCR were designed to span exon-exon junctions to minimize amplification of genomic DNA and their sequences can be found in Supplementary Table S2 (http://dx.doi.org/10.3168/jds.2015-9510). The housekeeping gene GAPDH was used as an internal control in these reactions.

\section{Statistical Analysis of Gene Expression}

Analysis of gene expression data and graph generation was completed using the software OriginLab (OriginLab Corporation, Northhampton, MA). For gene expression validation of selected miRNA, a paired $t$-test was used to assess differences of the mean $\Delta \mathrm{Ct}$ between degenerate and blastocyst conditioned media for each miRNA. Moreover, a paired $t$-test was used to assess expression differences of both miR-24 and $C D$ $K N 1 b$ within embryos cultured with or without mimic. For each analysis, significance was set as $P<0.05$.

\section{RESULTS}

\section{Analysis of Raw miRNA-Seq Data}

To assess the milieu of miRNA in in vitro culture media from embryos of differing quality, conditioned media was collected from both blastocyst and degenerate (an embryo which failed to develop from the morula to blastocyst stage) embryos for miRNA-seq. The media conditioned by embryos in addition to control SOF media (did not contain an embryo) was pooled and total RNA was extracted. A total of $68,948,165$ raw reads were obtained from miRNA-seq across all media samples. Total raw and mappable reads for each sample are listed in Supplementary Table S3 (http://dx.doi. org/10.3168/jds.2015-9510). Reads were filtered to remove those that did not contain a $3^{\prime}$ adapter and those that were less than 15 bases after $3^{\prime}$ adapter removal were removed. The raw sequences were then filtered once more to include those that were between 15 and 32 bases. A total of $58,870,746$ reads were obtained (Supplementary Figure S2 http://dx.doi.org/10.3168/ jds.2015-9510) and analyzed using the proprietary pipeline by LC Sciences (Houston, Texas). Analysis of the length distribution of reads showed that reads were of expected length for mature miRNA (17-22 nucleotides), where the greatest percentage $(28 \%)$ of mappable reads were 20 nucleotides in length (Figure 1). For the length distribution, unique miRNA and copy number of reads corresponding to each sequence length is illustrated in Supplementary Figure S3 (http://dx.doi.org/10.3168/ jds.2015-9510). Reads were either mapped to selected mir and miR in miRbase of both bovine and selected mammalian species, or were unmapped to selected mir in miRbase of both bovine and selected mammalian 


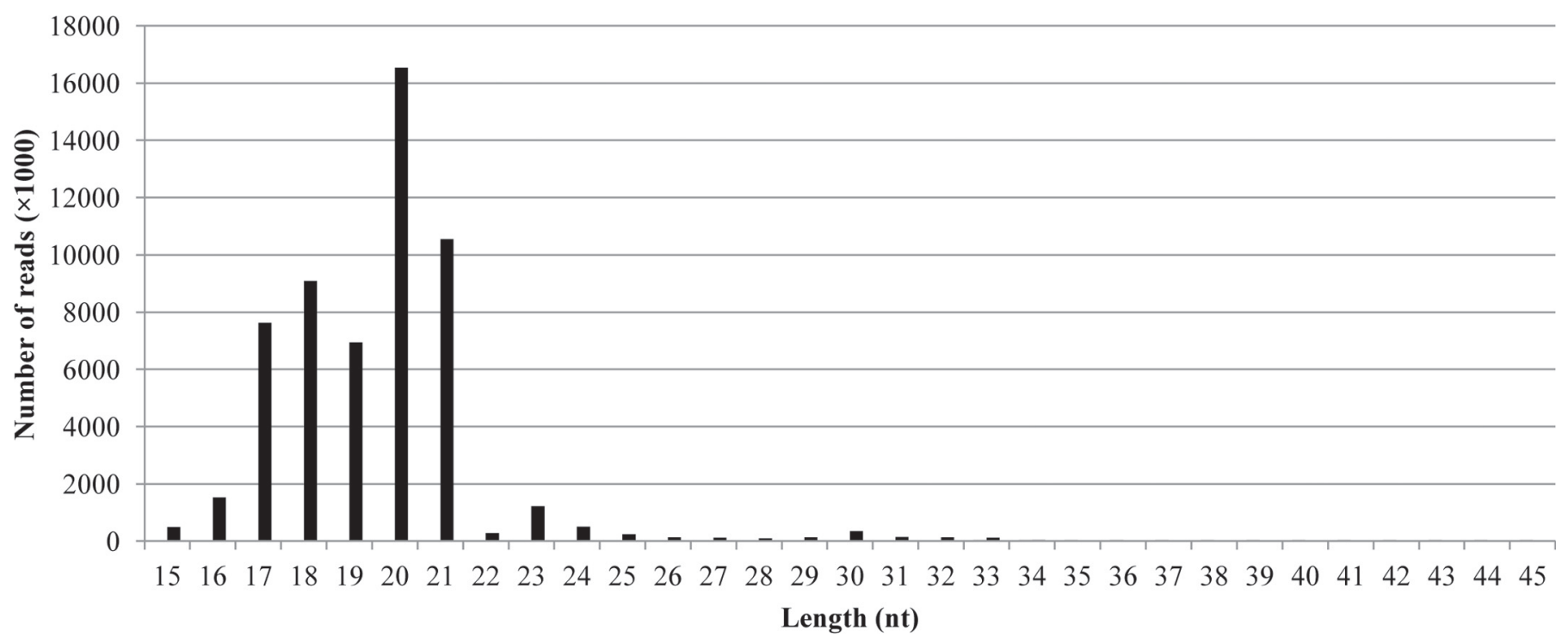

Figure 1. Length distribution of mappable reads.

species (Supplementary Figure S1). Using the program UNAFold, reads that were not mapped to known miR or mir were further categorized based on those that mapped to the bovine genome including other areas, such as mRNA and other RNA and that were likely to form a hairpin structure. Sequences were considered a "nohit" if they did not map to either miRbase, RepBase, RFam, or to the bovine reference genome.

Mapping of reads resulted in 22,623 sequences mapping to selected mir and miR in MiRbase. The distribution of mappable reads is illustrated in Figure 2. Interestingly, a large fraction of the reads mapped to mRNA in the culture media in addition to miRNA. A large proportion consisting of 40,394,271 mappable reads, were deemed "nohit," as these sequences did not map to either known mir or miR in MiRbase or other RNA sequences in RFam or Repbase. Of the mappable sequences, 385 mapped to known bovine miRNA (group 1a) and 87 mapped to known miR of selected mammalian species in miRbase (group 1b) but were considered novel to bovine (Table 1).Within selected mammalian species, 4 sequences mapped to known mir in addition to mapping to the bovine genome within a region predicted to form a hairpin secondary structure (group 2a) and 128 sequences mapped to known mir that mapped to the bovine genome but were not within a predicted hairpin (group 2b). A total of 508 sequences mapped to known mir and miR of selected species but did not map to the bovine genome (group 3a). In group 3b, 73 sequences were identified that did not map to miR but to mir of selected species and did not map to the bovine genome. An additional group of 211 sequences were unmappable to miRbase but were mapped to the bovine genome within regions that are predicted to form hairpins. Taken together, the sequencing data revealed the presence of known bovine miRNA, miRNA known in other mammalian species novel to bovine, as well as sequences that are previously unidentified bovine-specific miRNA.

\section{Differential Expression of miRNA in Culture Media}

Following mapping, the number of read copies for each sample was normalized for comparison of expression between blastocyst conditioned media, degenerate conditioned media, as well as the control SOF media, which was never in contact with an embryo but was cultured in the same petri dish. Differential expression is representative of the mean normalized reads followed by a paired $t$-test of data across media generated from 3 replicates of IVF experiments. Analysis revealed 11 miRNA that were differentially expressed between degenerate and blastocyst conditioned media (Table 2 ). The differentially expressed miRNA were all more highly expressed in degenerate media compared with blastocyst media and consisted of both known mir and miR specific to bovine and swine, and of predicted miR that mapped to the bovine genome and were within a predicted hairpin forming sequence. Between control SOF media and blastocyst conditioned media, 8 miRNA were differentially expressed and all were more highly expressed in control SOF media and were of either known bovine or swine sequences. Comparing control SOF media and degenerate conditioned media, 4 miRNA were differentially expressed, where 2 were expressed higher in degenerate media and 2 
Table 1. Classification of mappable sequences in in vitro fertilization culture media

\begin{tabular}{|c|c|c|}
\hline Item & Group & $\begin{array}{c}\text { Unique } \\
\text { microRNA, } \\
\text { no. }\end{array}$ \\
\hline Known microRNA of Bos taurus of selected species & $\begin{array}{l}\text { Group 1a } \\
\text { Group 1b }\end{array}$ & $\begin{array}{r}385 \\
87\end{array}$ \\
\hline \multicolumn{3}{|l|}{ Predicted microRNA } \\
\hline Mapped to known mature microRNA of selected species and genome; within hairpins & Group 2a & 4 \\
\hline Mapped to known mature microRNA of selected species and genome; no hairpins & Group 2b & 128 \\
\hline Overall (Unique pre-microRNA and mature microRNA) & & 1,244 \\
\hline
\end{tabular}

were expressed higher in the control SOF media (Table 2). Sequencing results of the control SOF media suggests though BSA, a known carrier of miRNA, was not supplemented to the media that other components in the media carry miRNA. However, morulae were transferred to the base SOF and differences in expression between blastocyst and degenerate media samples were detected and herein will be our focus.

\section{Gene Expression Validation}

To confirm the sequencing results, miRNA that were found to be significantly differentially expressed $(P<$
$0.05)$ between blastocyst and degenerate media were selected for validation of expression by qRT-PCR. The miRNA selected were mir-2887, miR-24, miR146a, miR-191, and miR-148a and were tested within new biological replicates of media pools generated in 3 additional IVF experiments. Expression analysis confirmed 3 miRNA, miR-24, miR-191, and miR-148a, to be more highly expressed in degenerate conditioned media compared with blastocyst conditioned media, whereas miR146a and miR-2887 tended to be differentially expressed $(P<0.07)$. The fold difference $( \pm \mathrm{SE})$ in expression between degenerate and blastocyst conditioned media for miR-2887, miR-24, miR-146a, miR-191, and miR-148a

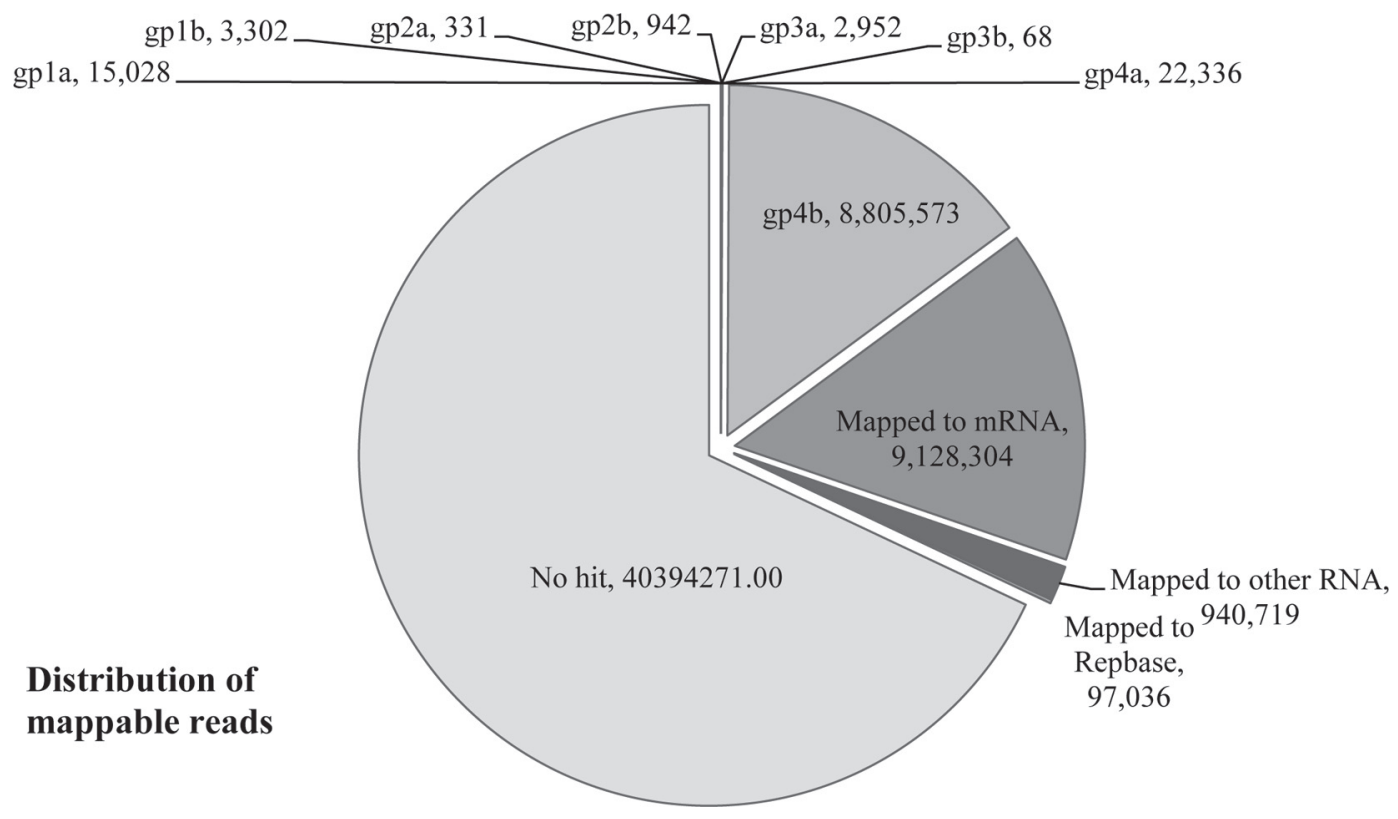

Figure 2. Distribution of mappable reads. gpla = known microRNA specific to bovine; gp1b = known microRNA of selected species, but novel to bovine; gp2a = mapped to known pre-microRNA of selected species and genome (within hairpins); gp2b = mapped to known premicroRNA of selected species and genome (no hairpins); gp3a = mapped to known pre-microRNA and mature microRNA of selected species but unmapped to bovine genome; gp3b = unmapped to known mature microRNA of selected species and unmapped to bovine genome; gp4a $=$ unmapped to known mature microRNA but mapped to bovine genome and within hairpins; gp4b = unmapped to known mature microRNA but mapped to genome and not within a predicted hairpin. 
Table 2. Differential expression of microRNA sequences in culture media

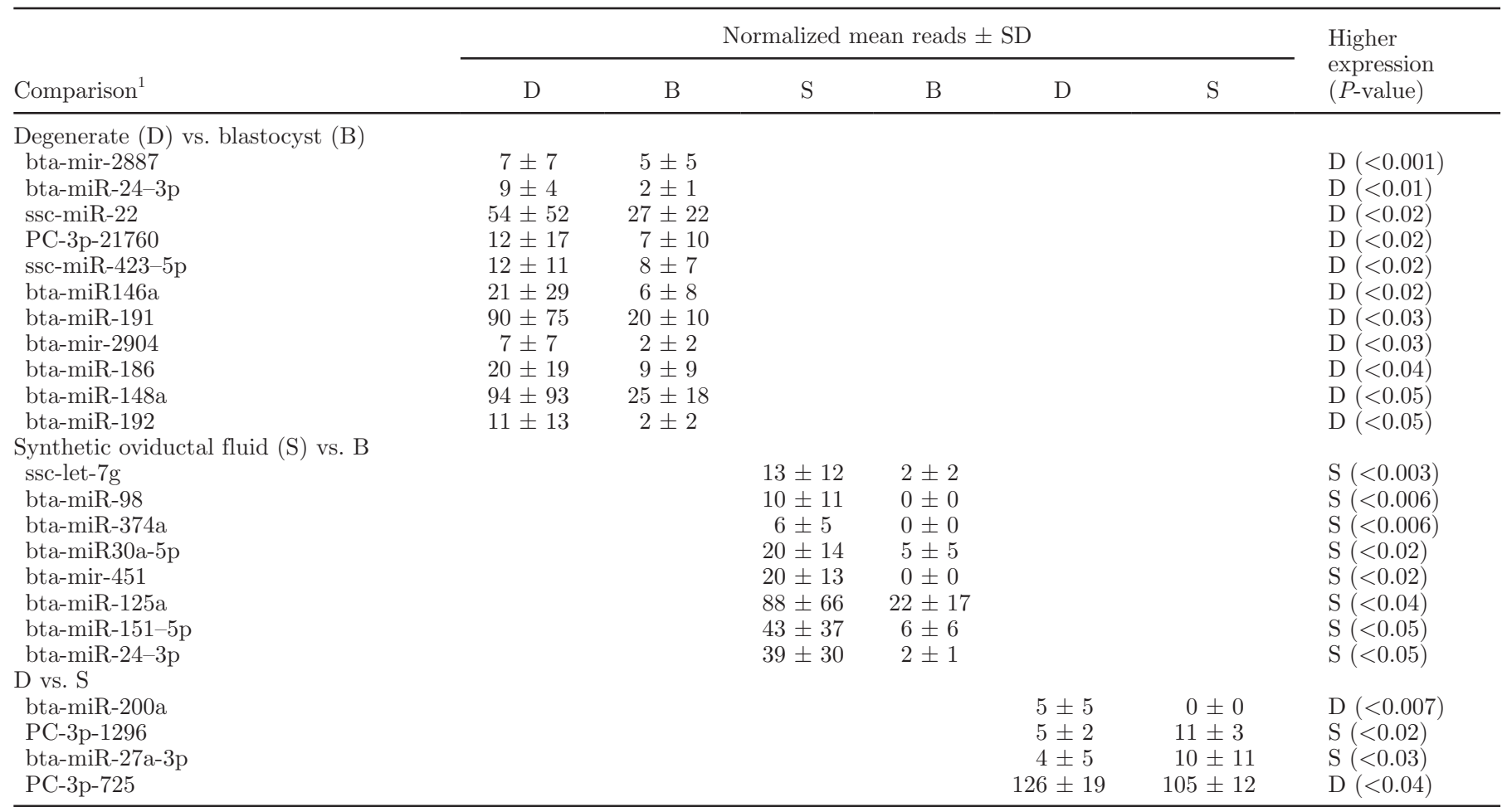

${ }^{1} \mathrm{PC}=$ sequences in Group 4a that are not in miRbase, map to the bovine genome, and are predicted to form hairpins; mir $=$ pre-microRNA; $\operatorname{miR}=$ mature microRNA.

was $7.4 \pm 2.4(P=0.07), 4.9 \pm 0.5(P<0.004), 2.4 \pm$ $0.5(P=0.06), 3.2 \pm 0.7(P<0.05)$, and $4.3 \pm 0.6(P$ $<0.05$ ), respectively (Figure 3 ). Thus, the expression of these miRNA detected by qRT-PCR confirms the miRNA-seq results.

\section{Supplementation of miR-24 to IVF Culture Media Reduces Embryonic Development and Represses its Target Gene CDKN1b}

To assess whether the presence of miRNA in the media affects embryonic development, a mimic of miR24 was supplemented to the IVF culture media on $\mathrm{d}$ 5 of development corresponding to the morula stage. Mature miRNA-24 was chosen as it was more highly expressed in degenerate and control media compared with blastocyst media, suggesting that the miRNA moves into the embryo or is degraded within the blastocyst media. Additionally, miR-24 is highly conserved across mammalian species and has been found to target mRNA relating to cell proliferation and apoptosis. The miR-24 mimic supplemented to the media was a synthetic double-stranded RNA, similar in nature to endogenous miRNA. Across 3 IVF replicates, morulastage embryos cultured in the presence of miR-24 mimic had a development rate of $48.3 \%$ compared with

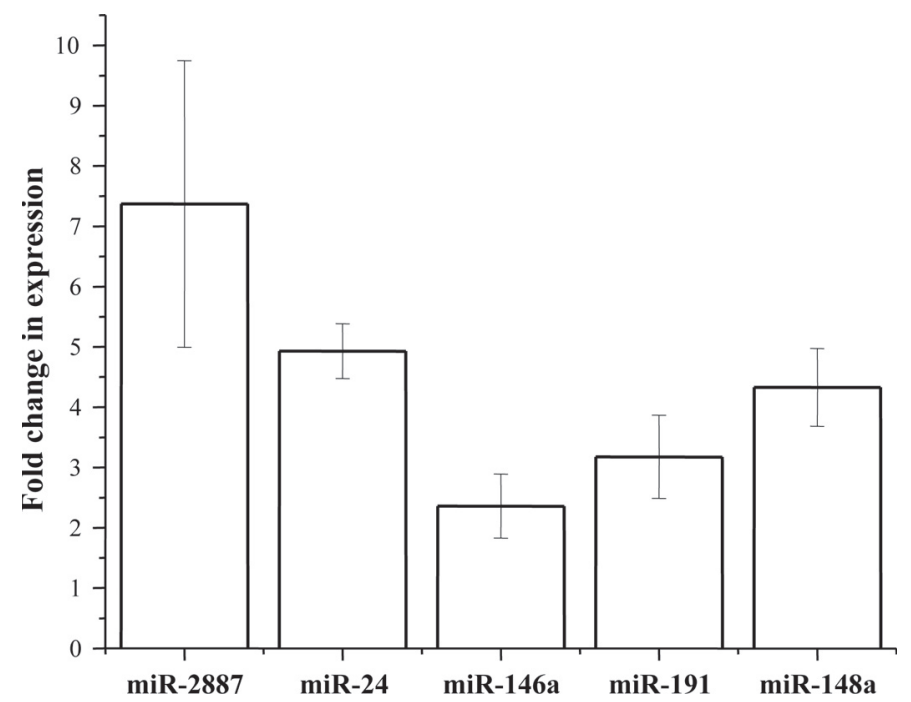

Figure 3. Gene expression validation of miRNA differentially expressed in degenerate media compared with blastocyst media. Error bars represent the expression range calculated as the fold change in expression using the SE of the mean fold change. Differential expression was confirmed for mature microRNA (miR)-24, miR-191, and miR-148a $(P<0.05)$, which were more highly expressed in degenerate media compared with blastocyst media, whereas miR-146a and miR2887 tended to be different $(P<0.07)$. 
Table 3. Mature microRNA (miR)-24 mimic supplementation to in vitro fertilization culture media reduces blastocyst development

\begin{tabular}{lcccc}
\hline Treatment group & $\begin{array}{c}\text { Total } \\
\text { morula }\end{array}$ & $\begin{array}{c}\text { Number of } \\
\text { blastocysts }\end{array}$ & $\begin{array}{c}\text { Number of } \\
\text { degenerates }\end{array}$ & $\begin{array}{c}\text { Morula developed } \\
\text { to blastocyst, \% }\end{array}$ \\
\hline Control & 156 & 118 & 38 & $75.6^{\mathrm{a}}$ \\
$1 \mu M$ miR-24 mimic & 149 & 72 & 77 & $48.3^{\mathrm{b}}$ \\
\hline a,b Differing superscripts within a column denote statistically significant differences $(P<0.0001)$.
\end{tabular}

controls where $75.6 \%$ of the morula developed to the blastocyst stage (Table 3). This significant decline in embryonic development $(P<0.0001$; chi-squared $)$ as a result of supplementation of synthetic miR-24 suggests that the extracellular miRNA milieu greatly affects the development of the embryo.

The miR-24 expression was profiled to understand expression across the stages of development in control embryos to better understand how alterations at differing time points may affect the embryo. Expression of miR-24 was significantly greater (fold change $=4.6$ $\pm 0.1, P<0.02$; one-way ANOVA) in morula-stage embryos in comparison to blastocyst-stage embryos. In contrast, the expression of miR-24 in degenerate embryos was highly variable, reflecting no significant differences between the morula or blastocyst stage. The expression profile suggests that miR-24 becomes downregulated from the morula stage to the blastocyst stage, where alterations in the gene regulation at this time point could lead to embryo growth arrest.

To better understand the mechanisms by which supplementation of mimic miR-24 affects embryonic development, expression levels of miR-24 and its target gene $C D K N 1 b$ were assessed in embryos cultured with the mimic. As illustrated in Figure 4, blastocyst embryos cultured in the presence of mimic miR-24 more highly expressed miR-24 by $44.3 \pm 11.8$ fold $(P<0.004)$ compared with control cultured blastocysts. Additionally, expression of a target mRNA of miR-24, CDKN1b, a cell cycle regulator, was significantly reduced in mimic blastocysts by $33 \%$ compared with that of control blastocysts (fold change $0.7 \pm 0.01 ; P<0.004$; Figure 4). Overall, increased presence of miR-24 in the extracellular environment leads to higher expression of miR-24 within embryos and results in decreased expression of a miR-24 mRNA target.

\section{DISCUSSION}

Previous studies identified miRNA present in the extracellular environment derived from varying cell types and have reported these molecules to be strong biomarkers of disease conditions. The present study aimed to further characterize the presence of miRNA in the culture media of IVF embryos for the potential to develop biomarkers of embryo quality in the future. The miRNA-seq detected miRNA to be differentially expressed in the media conditioned by embryos differing in developmental potential. To further assess the effect of extracellular miRNA on early embryonic development, addition of a mimic of miR-24 to the medium not only reduced development but also increased expression within the embryo and repressed expression of its target gene, $C D K N 1 b$, within embryos. A further understanding of the mechanisms and relationship between miRNA secreted and intracellular miRNA is warranted in the future. Here, the data set the framework for future work to establish a noninvasive genetic biomarker to survey embryos of similar morphology and to determine whether these markers are predictive of developmental potential in terms of pregnancy outcome.

Deep sequencing resulted in the detection of many miRNA sequences in the media. A large portion of the mappable miRNA was of known bovine miR, as expected with recent advances in annotating bovine

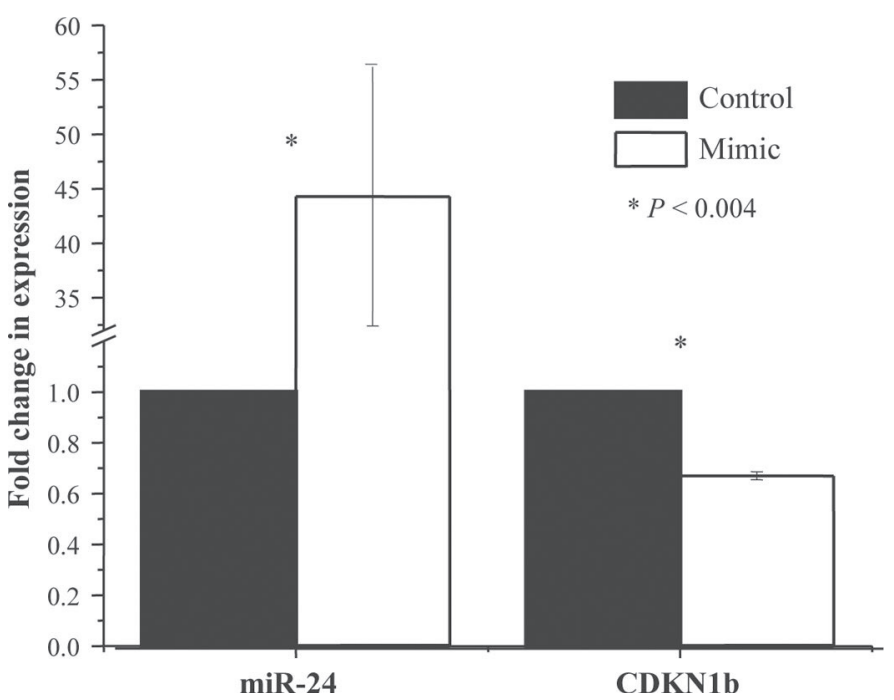

Figure 4. Mature microRNA (miR)-24 in the media increases mature microRNA-24 expression in embryos and represses its mRNA target, $C D K N 1 b$. Fold change in expression of mature microRNA-24 and $C D K N 1 b$ in control blastocysts versus mimic blastocysts. Error bars represent the expression range calculated as the fold change in expression using the SEM fold change. An asterisk denotes statistical significance $\left({ }^{*} P<0.004\right)$. 
miRNA. However, several other sequences mapped to both predicted and known mir and miR in other species, including swine and human matches, suggesting that further annotation of bovine miRNA is warranted. Indeed, a proportion of sequences detected in the media were not identified in miRbase but mapped to the bovine genome and were within sequences predicted to form hairpins. These sequences are potentially novel bovine sequences that are yet to be characterized in terms of annotation and function, including roles in embryonic development within culture media. Interestingly, these sequences were significantly detected across multiple IVF biological replicates, suggesting that they are not arbitrary to a particular IVF experiment and functionally are relevant to embryo development.

The miRNA spectrum greatly differs between degenerate and blastocyst conditioned media. Both Kropp et al. (2014) and Rosenbluth et al. (2014) have previously identified miRNA in the culture media, though the function of these miRNA in the extracellular environment is not known. Differences in the regulation of the miRNA within the embryo may be reflected in the extracellular milieu. Several of the miRNA identified as differentially expressed miRNA regulate genes associated with cellular proliferation. For example, overexpression of miR-146a is associated with an increase in cell proliferation in a pERK-dependent manner (Xu et al., 2012) and both miR-24 and miR-148a overexpression act to repress p27, thereby increasing cell proliferation (Guo et al., 2011; Giglio et al., 2013). Conversely, miR-24 overexpression in human cancer cells results in increased apoptosis by downregulation of $B C L-2$, an antiapoptotic gene (Srivastava et al., 2011). Thus, the antagonistic interplay of the mRNA targeted by miRNA within the embryo to promote or inhibit development is reflected by the miRNA present in media conditioned by embryos of differing quality. Additionally, miR-148a, a differentially expressed miRNA, may affect epigenetic regulation within the embryo. A previous study demonstrated that the interplay between miR-148a and its target DNMT1 forms a negative regulatory feedback loop, in which overexpression of DNMT1 leads to hypermethylation of miR-148a promoter, which in turn downregulates miR-148a expression (Xu et al., 2013b). Therefore, alterations in miRNA expression as a result of the IVF culture environment have the potential to alter embryonic development through epigenetic mechanisms such as DNA methylation.

MicroRNA present in the media may reflect abnormalities in development due to perturbations of the embryo's genetics. For example, both miR-146a and miR-191, which were more highly expressed in the degenerate media in the present study, have also been associated with embryonic abnormalities. Jeon et al. (2012) reported the genotypic combination of miR-146aCC and miR-196a2CC to be more frequent in chromosomally normal spontaneously aborted human fetuses. Similarly, Rosenbluth et al. (2014) found differential secretion into culture media of miR-191 by aneuploid embryos compared with normal euploid embryos. Determining whether the embryo's genomics, such as genotype or epigenetics, change the extracellular milieu, or conversely whether the extracellular environment induces changes to the embryo, needs further examination.

Further exploration of miR-24 expression revealed that there is a significant effect of extracellular miRNA on the developing embryo. The expression of miR-24 within embryos declines from the morula to the blastocyst stage. In the media, expression of miR-24 is lower in blastocyst-conditioned media compared with degenerate-conditioned media. Upon adding a greater concentration of miR-24 to the medium during the transition from the morula to blastocyst stage, development was significantly reduced, miR-24 expression increased within blastocyst embryos and resulted in reduced expression of its target mRNA, CDKN1b. It has been shown that overexpression of miR-24 represses $C D K N 1 b$, which is a cell cycle inhibitor, thereby promoting cell proliferation in several cancer cell lines (Giglio et al., 2013). In our study, cellular proliferation was not quantified; however, other likely targets of miR-24 may also be underlying cell proliferation and death. For instance, loss of miR-24 leads to upregulation of dihydrofolate reductase, an s-phase enzyme that regulates the cell cycle, and subsequent induction of neoplastic growth was observed in immortalized cell lines (Mishra et al., 2009). Other mRNA targets of miR-24 likely also play a role in poor development. Upregulation of miR-24 reduces the expression of $H 2 A X$ in terminally differentiated hematopoietic cells, which causes these cells to be overly sensitive to DNA damage, thereby leading to cellular demise (Lal et al., 2009). Moreover, exogenous overexpression of the miR cluster 23a-27 to 24 in bone suppressed osteoblast differentiation by repressing SAT2B, which acts with RUNX2 (Hassan et al., 2010). Thus, alterations of the level of miR-24 within the embryo as a response to extracellular miR24 concentration potentially perturb the expression of several key mRNA and gene networks regulating development.

Previously, researchers have demonstrated that serum supplementation to culture media provides an external source of miRNA (Turchinovich et al., 2011; Kropp et al., 2014; Rosenbluth et al., 2014). Irrespective of serum supplementation, several sequences were detected in the control SOF media, which was cultured in the same petri dish but did not contain nor came into contact 
with an embryo. As previously demonstrated by Kropp et al. (2014), miR-196a expression in culture media was linked to BSA supplementation of the culture media. Though serum was not supplemented to the culture medium from d 5 to 8 of development in our experiment, miRNA were still detected and likely to be as a result of other medium components. The base SOF medium components are considered proprietary by the manufacturer and components cannot be ruled out as potential carriers; however, other supplements are added that can be further tested in the future.

Gene expression changes observed in embryos cultured in the presence of a higher concentration of miR24 suggest that miRNA are taken up by these embryos from the media as the expression of miR-24 increases and functionally, a target mRNA is repressed. Initial RNA-sequencing revealed that miR-24 expression was higher in both degenerate and SOF control media in comparison to blastocyst media; however, no difference was observed between the baseline SOF control and degenerate media. Alone, the sequencing data suggests either up-take or degradation of miR-24 in blastocyst media. Although the control SOF media did in fact contain miRNA, the comparisons between blastocyst and degenerate media are still valid, as the base media is equally distributed to these embryos at d 5 in development and expression differences are reproducible. Differences in the detection of miRNA above or below the base SOF media could be as a result of secretion or uptake of miRNA from the extracellular environment. Studies have exemplified miRNA uptake into cells from the extracellular environment (Valadi et al., 2007; Sohel et al., 2013). Indeed a study by Sohel et al. (2013) demonstrated uptake of exosome-coupled miRNA from bovine follicular fluid into granulosa cells with a corresponding increase in endogenous miRNA in transfected cells and downregulation of target genes of the exosome-coupled miRNA.

The study by Sohel et al. (2013) also demonstrates the role miRNA have in cell-to-cell signaling. In our study, miR-200a concentration was higher in degenerate media compared with the SOF control as well as blastocyst media. Interestingly, studies reported that miR-200a present in both human myometrial cells and in mice uterine luminal epithelial cells can alter the uterine environment by repressing STAT5, which subsequently induces the expression of the progesterone

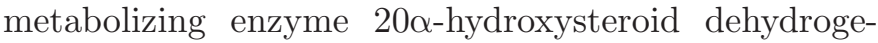
nase (Williams et al., 2012; Haraguchi et al., 2014). As this miRNA is secreted by a degenerate embryo, it could potentially act as a maternal recognition factor to alter progesterone levels signaling the presence of an abnormal embryo thereby preventing implantation.
Characterization of the function of these miRNA at the maternal-fetal interface is warranted in the future.

\section{CONCLUSIONS}

In conclusion, the present study demonstrates variation in the miRNA spectra within media conditioned by embryos of differing developmental competence. Though mechanisms of miRNA trafficking into and out of the embryo are not well defined, the expression data suggests miRNA are taken up by the embryo creating differences between embryos of differing quality. MicroRNA molecules are complex in nature, as they regulate multiple target mRNA; thus, supplementation of external sources of miRNA into the medium could alter embryonic development and should be further investigated. Differences in miRNA expression within media derived from embryos of differing developmental competence allows for future development of biomarkers for selecting better quality embryos in terms of ability to establish a successful pregnancy.

\section{ACKNOWLEDGMENTS}

Support for this research was provided by the University of Wisconsin-Madison Graduate School with funding from the Wisconsin Alumni Research Foundation. The authors thank Alyssa Daniels, an undergraduate student at the University of Wisconsin-Madison, for assisting in IVF experiments.

\section{REFERENCES}

Ambros, V. 2004. The functions of animal microRNAs. Nature 431:350-355.

Bartel, D. P. 2004. MicroRNAs: Genomics, biogenesis, mechanism, and function. Cell 116:281-297.

Bo, G. A., and R. J. Mapletoft. 2013. Evaluation and classification of bovine embryos. Anim. Reprod. 10:344-348.

Chandra, A., C. Copen, and E. Stephen. 2014. Infertility and Impaired Fecundity in the U.S.-1982-2010 Data from the National Health Survey of Family Growth. United States Centers for Disease Control and Prevention, Atlanta, GA.

Chen, X., Y. Ba, L. J. Ma, X. Cai, Y. Yin, K. H. Wang, J. G. Guo, Y. J. Zhang, J. N. Chen, X. Guo, Q. B. Li, X. Y. Li, W. J. Wang, Y. Zhang, J. Wang, X. Y. Jiang, Y. Xiang, C. Xu, P. P. Zheng, J. B. Zhang, R. Q. Li, H. J. Zhang, X. B. Shang, T. Gong, G. Ning, K. Zen, J. F. Zhang, and C. Y. Zhang. 2008. Characterization of microRNAs in serum: A novel class of biomarkers for diagnosis of cancer and other diseases. Cell Res. 18:997-1006.

Diskin, M. G., and D. G. Morris. 2008. Embryonic and early foetal losses in cattle and other ruminants. Reprod. Domest. Anim. 43:260-267.

Diskin, M. G., M. H. Parr, and D. G. Morris. 2012. Embryo death in cattle: An update. Reprod. Fertil. Dev. 24:244-251.

Driver, A. M., W. Huang, J. Kropp, F. Penagaricano, and H. Khatib. 2013. Knockdown of CDKN1C (p57(kip2)) and PHLDA2 results in developmental changes in bovine pre-implantation embryos. PLoS ONE 8:e69490. 
Driver, A. M., F. Penagaricano, W. Huang, K. R. Ahmad, K. S. Hackbart, M. C. Wiltbank, and H. Khatib. 2012. RNA-Seq analysis uncovers transcriptomic variations between morphologically similar in vivo- and in vitro-derived bovine blastocysts. BMC Genomics $13: 118$.

Farh, K. K. H., A. Grimson, C. Jan, B. P. Lewis, W. K. Johnston, L. P. Lim, C. B. Burge, and D. P. Bartel. 2005. The widespread impact of mammalian microRNAs on mRNA repression and evolution. Science 310:1817-1821.

Farin, P. W., J. A. Piedrahita, and C. E. Farin. 2006. Errors in development of fetuses and placentas from in vitro-produced bovine embryos. Theriogenology 65:178-191.

Filipowicz, W., S. N. Bhattacharyya, and N. Sonenberg. 2008. Mechanisms of post-transcriptional regulation by microRNAs: Are the answers in sight? Nat. Rev. Genet. 9:102-114.

Giglio, S., R. Cirombella, R. Amodeo, L. Portaro, L. Lavra, and A. Vecchione. 2013. MicroRNA miR-24 promotes cell proliferation by targeting the CDKs inhibitors p27Kip1 and p16INK4a. J. Cell. Physiol. 228:2015-2023.

Guo, S. L., Z. Peng, X. Yang, K. J. Fan, H. Ye, Z. H. Li, Y. Wang, X. L. Xu, J. Li, Y. L. Wang, and Y. Teng. 2011. miR-148a promoted cell proliferation by targeting p27 in gastric cancer cells. Int. J. Biol. Sci. 7:567-574.

Haraguchi, H., T. Saito-Fujita, Y. Hirota, M. Egashira, L. Matsumoto, M. Matsuo, T. Hiraoka, K. Koga, N. Yamauchi, M. Fukayama, A. Bartos, J. Cha, S. K. Dey, T. Fujii, and Y. Osuga. 2014. MicroRNA-200a locally attenuates progesterone signaling in the cervix, preventing embryo implantation. Mol. Endocrinol. 28:1108-1117.

Hassan, M. Q., J. A. R. Gordon, M. M. Beloti, C. M. Croce, A. J. van Wijnen, J. L. Stein, G. S. Stein, and J. B. Lian. 2010. A network connecting Runx2, SATB2, and the miR-23a similar to 27a similar to 24-2 cluster regulates the osteoblast differentiation program. Proc. Natl. Acad. Sci. USA 107:19879-19884.

Huang, W., B. S. Yandell, and H. Khatib. 2010. Transcriptomic profiling of bovine IVF embryos revealed candidate genes and pathways involved in early embryonic development. BMC Genomics 11:23.

Jeon, Y. J., S. Y. Kim, H. Rah, D. H. Choi, S. H. Cha, T. K. Yoon, W. S. Lee, S. H. Shim, and N. K. Kim. 2012. Association of the miR$146 \mathrm{aC}>\mathrm{G}, \operatorname{miR}-149 \mathrm{~T}>\mathrm{C}, \operatorname{miR}-196 \mathrm{a} 2 \mathrm{~T}>\mathrm{C}$, and miR-499A $>$ $\mathrm{G}$ polymorphisms with risk of spontaneously aborted fetuses. Am. J. Reprod. Immunol. 68:408-417.

Kropp, J., S. M. Salih, and H. Khatib. 2014. Expression of microRNAs in bovine and human pre-implantation embryo culture media. Front. Genet. 5:91.

Lal, A., Y. F. Pan, F. Navarro, D. M. Dykxhoorn, L. Moreau, E. Meire, Z. Bentwich, J. Lieberman, and D. Chowdhury. 2009. miR24-mediated downregulation of $\mathrm{H} 2 \mathrm{AX}$ suppresses DNA repair in terminally differentiated blood cells. Nat. Struct. Mol. Biol. 16:492-498.

Lim, L. P., N. C. Lau, P. Garrett-Engele, A. Grimson, J. M. Schelter, J. Castle, D. P. Bartel, P. S. Linsley, and J. M. Johnson. 2005. Microarray analysis shows that some microRNAs downregulate large numbers of target mRNAs. Nature 433:769-773.

Livak, K. J., and T. D. Schmittgen. 2001. Analysis of relative gene expression data using real-time quantitative PCR and the $2(\mathrm{~T})$ (-Delta Delta C) method. Methods 25:402-408.

Lucy, M. C. 2001. Reproductive loss in high-producing dairy cattle: Where will it end? J. Dairy Sci. 84:1277-1293.

Mishra, P. J., B. Song, Y. Wang, R. Humeniuk, D. Banerjee, G. Merlino, J. F. Ju, and J. R. Bertino. 2009. MiR-24 tumor suppressor activity is regulated independent of p53 and through a target site polymorphism. PLoS ONE 4:e8445.

Mitchell, P. S., R. K. Parkin, E. M. Kroh, B. R. Fritz, S. K. Wyman, E. L. Pogosova-Agadjanyan, A. Peterson, J. Noteboom, K. C. O'Briant, A. Allen, D. W. Lin, N. Urban, C. W. Drescher, B. S Knudsen, D. L. Stirewalt, R. Gentleman, R. L. Vessella, P. S. Nelson, D. B. Martin, and M. Tewari. 2008. Circulating microRNAs as stable blood-based markers for cancer detection. Proc. Natl. Acad. Sci. USA 105:10513-10518.
Parrish, J. J., A. Krogenaes, and J. L. Suskoparrish. 1995. Effect of bovine sperm separation by either swim-up or percoll method on success of in vitro fertilization and early embryonic-development. Theriogenology 44:859-869.

Rayner, K. J., and E. J. Hennessy. 2013. Extracellular communication via microRNA: Lipid particles have a new message. J. Lipid Res. $54: 1174-1181$

Rosenbluth, E. M., D. N. Shelton, L. M. Wells, A. E. T. Sparks, and B. J. Van Voorhis. 2014. Human embryos secrete microRNAs into culture media-A potential biomarker for implantation. Fertil. Steril. 101:1493-1500.

Santos, J. E., W. W. Thatcher, R. C. Chebel, R. L. Cerri, and K. N. Galvao. 2004. The effect of embryonic death rates in cattle on the efficacy of estrus synchronization programs. Anim. Reprod. Sci. 82-83:513-535.

Sohel, M. M. H., M. Hoelker, S. S. Noferesti, D. Salilew-Wondim, E. Tholen, C. Looft, F. Rings, M. J. Uddin, T. E. Spencer, K. Schellander, and D. Tesfaye. 2013. Exosomal and non-exosomal transport of extra-cellular microRNAs in follicular fluid: Implications for bovine oocyte developmental competence. PLoS ONE 8:e78505

Srivastava, N., S. Manvati, A. Srivastava, R. Pal, P. Kalaiarasan, S. Chattopadhyay, S. Gochhait, R. Dua, and R. N. K. Bamezai. 2011. miR-24-2 controls H2AFX expression regardless of gene copy number alteration and induces apoptosis by targeting antiapoptotic gene BCL-2: A potential for therapeutic intervention. Breast Cancer Res. 13:R39.

Turchinovich, A., L. Weiz, A. Langheinz, and B. Burwinkel. 2011. Characterization of extracellular circulating microRNA. Nucleic Acids Res. 39:7223-7233.

Valadi, H., K. Ekstrom, A. Bossios, M. Sjostrand, J. J. Lee, and J. O. Lotvall. 2007. Exosome-mediated transfer of mRNAs and microRNAs is a novel mechanism of genetic exchange between cells. Nat. Cell Biol. 9:654-659.

Van Soom, A., B. Mateusen, J. Leroy, and A. De Kruif. 2003. Assessment of mammalian embryo quality: What can we learn from embryo morphology? Reprod. Biomed. Online 7:664-670.

Vandesompele, J., K. De Preter, F. Pattyn, B. Poppe, N. Van Roy, A. De Paepe, and F. Speleman. 2002. Accurate normalization of real-time quantitative RT-PCR data by geometric averaging of multiple internal control genes. Genome Biol. 3:R0034.

Wang, K., S. L. Zhang, J. Weber, D. Baxter, and D. J. Galas. 2010. Export of microRNAs and microRNA-protective protein by mammalian cells. Nucleic Acids Res. 38:7248-7259.

Wang, Z., H. Yao, S. Lin, X. Zhu, Z. Shen, G. Lu, W. S. Poon, D. Xie, M. C. M. Lin, and H. F. Kung. 2013. Transcriptional and epigenetic regulation of human microRNAs. Cancer Lett. 331:1-10.

Williams, K. C., N. E. Renthal, J. C. Condon, R. D. Gerard, and C. R. Mendelson. 2012. MicroRNA-200a serves a key role in the decline of progesterone receptor function leading to term and preterm labor. Proc. Natl. Acad. Sci. USA 109:7529-7534.

Xu, B., N. Wang, X. H. Wang, N. Tong, N. Shao, J. Tao, P. C. Li, X. B. Niu, N. H. Feng, L. H. Zhang, L. X. Hua, Z. J. Wang, and M. Chen. 2012. MiR-146a suppresses tumor growth and progression by targeting EGFR pathway and in a p-ERK-dependent manner in castration-resistant prostate cancer. Prostate 72:1171-1178.

Xu, L., B. F. Yang, and J. Ai. 2013a. MicroRNA transport: A new way in cell communication. J. Cell. Physiol. 228:1713-1719.

Xu, Q., Y. Jiang, Y. Yin, Q. Li, J. He, Y. Jing, Y. T. Qi, W. Li, B. Lu, S. S. Peiper, B. H. Jiang, and L. Z. Liu. 2013b. A regulatory circuit of miR-148a/152 and DNMT1 in modulating cell transformation and tumor angiogenesis through IGF-IR and IRS1. J. Mol. Cell Biol. 5:3-13.

Zhang, B., F. Penagaricano, H. Chen, and H. Khatib. 2012. Novel transcripts and alternatively spliced genes are associated with early development in bovine embryos. Animal 6:1199-1205. 